\title{
Microwave application for the detection of biodiesel-glycerine and biodiesel-water interfaces in the biodiesel production
}

\begin{abstract}
In biodiesel industry there is a need to detect interfacing layer between biodiesel and glycerine after the transesterification and interfacing layer between water and biodiesel after the washing process. The detection of these two interfaces is important for separation process. In this work, a simple, low cost, and accurate microwave reflection type system for production of coconut biodiesel has been applied for the detection these interfaces. A module that works at $10.7 \mathrm{GHz}$, consisting of a microwave generator and a detecting diode, is used to measure the microwave reflection coefficient at various positions through the tank. The experimental results show the ability of the system to detect the level or height of these interfaces at the accuracy of $0.1 \mathrm{~mm}$.
\end{abstract}

Keyword: Biodiesel-glycerine; Biodiesel-water; Microwave reflection 